\title{
Time-Frequency Analysis during Continuous Incremental Isometric Exercise
}

\author{
MC Mántaras ${ }^{1,2}$, S Carrasco Sosa ${ }^{3}$, A Guillén Mandujano ${ }^{3}$, R González Camarena ${ }^{3}$, MJ Gaitán \\ González ${ }^{3}$ \\ ${ }^{1}$ Departamento Ingeniería Eléctrica - DCBI - UAMI - México \\ ${ }^{2}$ Departamento Bioingeniería - FI - UNER - Argentina \\ ${ }^{3}$ Departamento de Ciencias de la Salud - DCBS - UAMI - México
}

\begin{abstract}
Few authors have used spectral analysis of heart rate variability to characterize cardiovascular control mechanisms during isometric exercise. This technique requires stationary signals and long exercise protocols for different loads, using at least three minutes per load. In this work we explore the use of cardiovascular timevariant spectral indexes during a continuous incremental isometric exercise protocol. We studied the effect of three exercise loads for isometric one leg-knee extension on cardiovascular response. Two respiratory conditions, spontaneous and controlled breathing, were evaluated. Born-Jordan time-frequency distribution was used to estimate time-variant power spectra from RR intervals, systolic pressure and breathing series. The use of timevariant analysis allowed us to quantify cardiovascular spectral indexes and to discriminate among three continuous exercise loads using a very short-term protocol of isometric exercise.
\end{abstract}

\section{Introduction}

Isometric exercise (IE) is characterized by increments in both arterial pressure and heart rate. Cardiovascular response to exercise involves changes in autonomic nervous system (ANS) activity. It is currently assumed that three neural mechanisms are concerned in this response [1]-[2][5]: the central command, the muscle metaboreflex, and the arterial baroreceptor reflex.

Few works in literature [6][7] have used spectral analysis to characterize cardiovascular control mechanisms during one leg isometric exercise.

Methods for the spectral analysis of heart rate variability (HRV) have been used and studied as a noninvasive alternative technique with purpose of evaluating the physiological behavior of the cardiovascular control system. It was found that high frequency component (HF), between 0.15 and $0.5 \mathrm{~Hz}$, describes parasympathetic activity of autonomic nervous system, while that between 0.04 and $0.15 \mathrm{~Hz}$, named low frequency component (LF), gives an idea of sympathetic modulation [8]-[9].

Conventional spectral analysis assumes stationarity and ergodicity of signals, characteristics that are not present in the cardiovascular response to exercise. In addition, this technique requires long exercise protocols for different loads, using at least three minutes per load. However, there are various approaches that can overcome the problem of non stationarity, such as time-frequency distributions, wavelets and time-varying autoregressive analysis [10]-[14].

In this work, we explore the use of a time-variant spectral analysis in order to evaluate cardiovascular response during a short-term continuous incremental isometric exercise protocol under spontaneous and controlled breathing.

\section{Methods}

Ten healthy volunteers, aged $23.25 \pm 3.50$ years, participated in this study. All participants gave their written informed consent. All were non-smokers and not involved in regular physical activity.

Isometric exercise maneuver consisted of 1 leg-knee extension (ILE) in seated position. Exercise maneuver was performed in three consecutive one-minute loads of ILE at $15 \%(\mathrm{E} 1), 30 \%(\mathrm{E} 2)$ and $45 \%(\mathrm{E} 3)$ of maximum voluntary contraction (MVC). MVC was determined as highest force developed by the subjects in three trials, each one 3 seconds long.

Protocol consisted in two conditions, spontaneous and controlled breathing. A visual feedback allowed subjects to follow respiratory stimulus and maintain respiratory control at $0.2 \mathrm{~Hz}$ and 0.8 liters.

Each condition consisted of 3 stages: control, oneminute at rest; three-minute exercise maneuver, three consecutive loads; and two-minute of recovery at rest.

Studies were performed in the morning, and subjects were summoned two times, one per each respiratory condition. Subjects were required not to eat or drink coffee at least 2 hours before the study. 
Electrocardiogram (ECG), blood pressure, respiratory volume and extension force were continuously registered and acquired (Biopac System). To record ECG, the CM5 electrocardiographic lead was processed through Biopac ECG Amplifier. Non-invasive arterial pressure was continuously measured at the middle finger of nondominant hand by Finapres (Ohmeda, model 2300). The arm with the instrumented finger was held extended at heart level by means of a sling. Respiration was assessed by a pneumotachometer (Hans Rudolph 2700) and a pressure transducer set (Validyne CD19A) connected to a face mask. Respiratory volume was obtained by integration of respiratory flow (Validyne FV156 Integrator). Extension force was measured by a Smedley hand dynamometer (Stoelting 56380), appropriately adapted, and registered through Biopac Amplifier. The 4 analog signals were acquired at $500 \mathrm{~Hz}$ per channel, and stored in a personal computer for offline analysis.

RR intervals, systolic pressure (SP) and breathing series were extracted offline from acquired signals. Systolic pressure and respiratory series were obtained from respective signals sampled with the occurrence of $\mathrm{R}$ wave in ECG. Mean and standard deviation were computed for all series.

Prior to perform spectral analysis, series were resampled at $4 \mathrm{~Hz}$ and detrended [15]. Born-Jordan timefrequency distribution was used to estimate time-variant power spectra from RR intervals, systolic pressure and breathing series. High (HF; $0.15-0.5 \mathrm{~Hz})$ and low frequency (LF; $0.04-0.15 \mathrm{~Hz}$ ) components were computed from the obtained power spectra. For statistical analysis, we considered six one-minute stages: control, exercise maneuver (E1, E2, E3), early recovery (R1) and late recovery (R2). For all statistical comparisons, ANOVA for repeated measurements and post hoc Bonferroni test were used $(\mathrm{P}<0.05)$.

\section{Results}

In both spontaneous and controlled breathing, we observed the well-known behavior of cardiovascular system during exercise, decrease of RR intervals and increase of systolic arterial pressure. Mean of RR intervals, rMSSD and mean systolic arterial pressure are shown in figure 1. RR intervals (figure 1 - top) decreased progressive and continuously for incremental exercise loads, returning to control levels at recovery. In relation to control stage, this reduction was significant $(\mathrm{P}<0.05)$ for each exercise maneuver stage (E1, E2 and E3).

The rMSSD (figure 1 - middle) presented a similar behavior to RR intervals. However, reduction was just significant for E2 and E3.
Mean SP series (figure 1 - bottom) raised with increasing exercise loads, and returned to control level during recovery. This augmentation was significant for E2 and E3 under spontaneous breathing and for E3 just in controlled breathing condition.

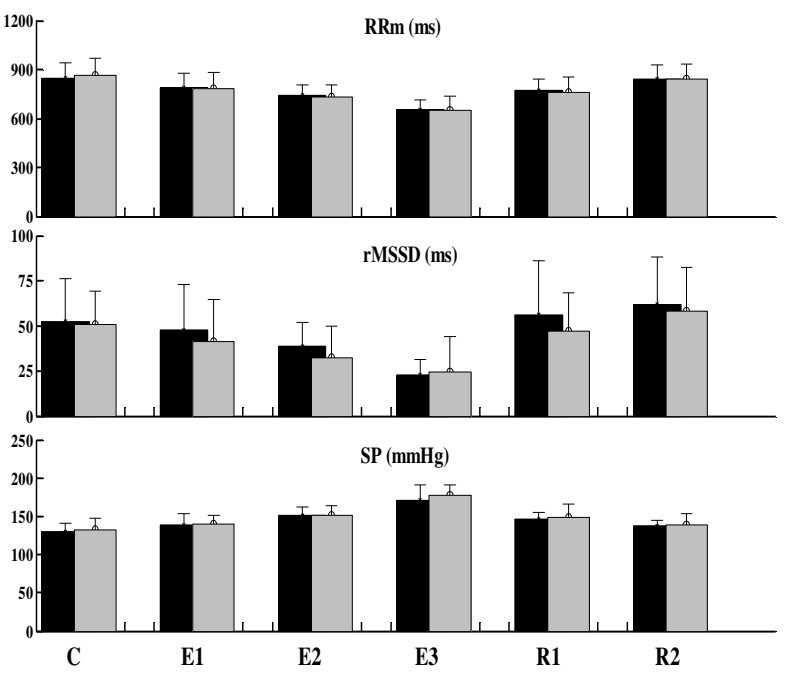

Figure 1. Mean and standard deviation (SD) of RR intervals, rMSSD and SP. Bars denote mean values (spontaneous breathing - black; controlled breathing grey) and lines indicate SD for each protocol stage. ' $\mathrm{C}$ ' control, 'E1' exercise (15\% MVC), 'E2' exercise (30\% MVC), 'E3' exercise (45\% MVC), 'R1' early recovery, and ' $\mathrm{R} 2$ ' late recovery.

Spectral densities of RR intervals obtained by BornJordan distribution are depicted in figure 2. In spontaneous breathing condition, more spectral components dispersion was observed. Under controlled breathing condition the respiratory component, centered in $0.2 \mathrm{~Hz}$, was evident.

Spectral indexes for RR intervals are illustrated in figure 3. For both studied respiratory conditions, in relation to control stage, RR intervals' total power (TP, figure 3 - top) and high frequency component (HF, figure 3 - bottom), progressively decreased along the increments of the exercise loads, while increased during recovery. This decrement was significant for E2 and E3 stages under both studied breathing conditions. LF component (figure 3 - middle) also decreased in E3, for spontaneous breathing condition. In general, under spontaneous breathing condition, the spectral indexes presented higher variance than under controlled breathing. 

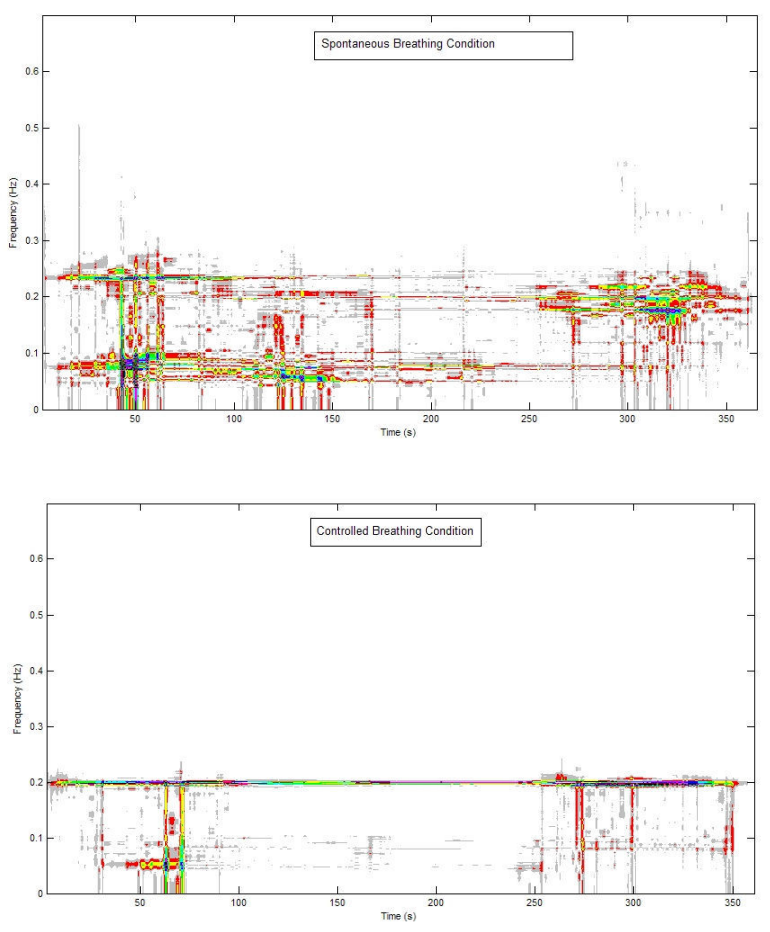

Figure 2. Spectral Densities obtained by Born Jordan distribution under the two protocol conditions, Spontaneous Breathing (top) \& Controlled Breathing (bottom).

\section{Discussion and conclusions}

Mean of RR intervals and systolic arterial pressure presented the expected behavior for an incremental exercise protocol. Reduction of rMSSD is consistent with vagal withdraw during exercise.

In general, spectral components presented higher variance in spontaneous breathing condition, possibly due to respiratory adjustments during exercise. When respiration was controlled, frequency components became more regular.

LF component behavior along increasing exercise intensity was ambiguous. For both considered respiratory conditions, HF component behavior was more obvious, showing a progressive decrease with increasing exercise loads, indicating gradual vagal withdraw. In this sense, HF component adequately indicates autonomic behavior during IE. Conversely, LF component did not display a gradual change for progressively increasing exercise intensity.
The assessment of HRV with traditional spectral analysis techniques during increasing intensity IE (3 or 4 minutes per load) results very difficult, due to the fast muscular fatigue which is characteristic of this exercise.
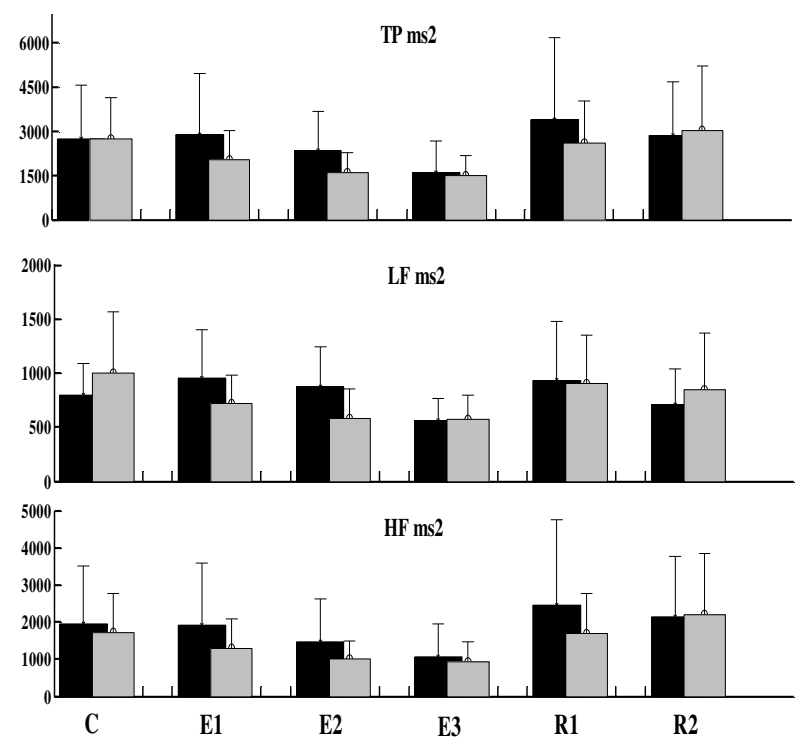

Figure 3. Spectral indexes for RR intervals. Bars denote mean values and lines standard deviation for each protocol stage. ' $\mathrm{C}$ ' control, 'E1' exercise (15\% MVC), 'E2' exercise (30\% MVC), 'E3' exercise (45\% MVC), ' $\mathrm{R} 1$ ' early recovery, and ' $\mathrm{R} 2$ ' late recovery.

Our preliminary results show that this difficulty can be overcome by means of a short-term IE protocol, where intensity is progressively augmented every minute, allowing several loads in the same maneuver. However, this approach generated non-stationary signals. A timefrequency distribution allows us to satisfactorily estimate HRV spectral density in this non-stable state. In this way, the use of time-variant analysis permitted us to quantify cardiovascular spectral indexes to discriminate between three continuous exercise loads, under two breathing conditions. Hence, it was possible to evaluate autonomic nervous activity during an incremental IE protocol.

In conclusion, time-variant techniques are appropriate tools to characterize, during short-term protocols, changes in cardiovascular variables during continuous incremental isometric exercise. 


\section{References}

[1] Goodwin GM, McCloskey DI \& Mitchell JH "Cardiovascular and respiratory responses to changes in central command during isometric exercise at constant muscle tension.” J. Physiol. (London), 1972; 226:173-90.

[2] Mitchell JH "Cardiovascular control during exercise: central and reflex neural mechanisms" Am. J. Cardiol. 1985; 55:D34-D41.

[3] O'Leary DS “Autonomic mechanisms of muscle metaboreflex control of heart rate" J. Appl. Physiol. 1993; 74:1748-54.

[4] Rowell LB "Human cardiovascular control" Oxford Univ. Press, New York. 1993.

[5] Iellamo F "Neural mechanisms of cardiovascular regulation during exercise" Auton Neurosci: Basic and clinical, 2001; 90:66-75.

[6] Iellamo F, Pizzinelli P, Massaro M, Raimondi G, Peruzzi G, Legramante JM "Muscle Metaboreflex Contribution to Sinus Node Regulation During Static Exercise. Insights From Spectral Analysis of Heart Rate Variability" Circulation. 1999; 100:27-32.

[7] Gonzalez-Camarena R, Carrasco-Sosa S, Roman-Ramos R, Gaitan-Gonzalez MJ, Medina-Bañuelos V, Azpiroz-Leehan $\mathrm{J}$ "Effect of static and dynamic exercise on heart rate and blood pressure variabilities" Med Sci Sports Exerc. 2000; 32(10):1719-28.

[8] Berntson GG, Bigger JT Jr., Eckberg DL, Grossman P, Kaufmann PG, Malik M and Van Der Molen MW "Heart rate variability: Origins, methods, and interpretive caveats," Psychophysiology, 1997; 34:623-48.
[9] Task Force of the European Society of Cardiology, and the North America Society of Pacing and Electrophysiology, "Heart rate variability standards of measurement, physiological interpretation and clinical use," Circulation, 1996; 93:1043-65.

[10] Cohen L. "Time-frequency distribution: A review". Proc IEEE, 1989; 77:940-81.

[11] Poularikas AD, "The transform and applications handbook." Huntsville, Alabama: IEEE Press, 1995; 887962.

[12] Marple S, "Digital spectral analysis with applications," Ed. Englewood Cliffs, NJ: Prentice-Hall, 1987.

[13] Chui C., "Wavelets, a tutorial in theory and applications" Academic Press, NY, 1992.

[14] Cerutti S, Bianchi A \& Mainardi L "Advanced spectral methods for detecting dynamic behavior" Auton Neurosci: Basic and clinical , 200; 90:3-12.

[15] Tarvainen MP, Ranta-aho PO, \& Karjalainen. "An advanced detrending method with application to HRV analysis”. IEEE Trans. Biomed. Eng. 2002; 42(2):172-174.

Address for correspondence

María Carla Mántaras

Departamento de Bioingeniería - Facultad de Ingeniería - UNER

C.C. 57 - Suc. 3, E3100, Paraná, Entre Ríos, Argentina

mailto: cmantaras@bioingenieria.edu.ar 